\title{
Liste des tables
}

Table $2.1 \quad$ Nomenclature I 21

Table $2.2 \quad$ Nomenclature II -22

Table $2.3 \quad$ Nomenclature III -23

Table $2.4 \quad$ Nomenclature IV -24

Table $2.5 \quad$ Nomenclature $\mathrm{V} \longrightarrow \mathbf{2 7}$

Table 4.1 Système des voyelles du protoroman, cf. Gouvert $(2014,70)-228$

Table 4.2 Système des consonnes du protoroman, adapté de Gouvert $(2014,118) \longrightarrow \mathbf{2 2 8}$

Table 4.3 Notation $\langle\mathrm{u}\rangle \sim\langle\mathrm{v}\rangle \mathrm{vs.} * / \beta /-229$

Table 4.4 Quatre cas flagrants de non-correspondance entre étymons « latinisants » et étymons reconstruits - 229

Table 4.5 Reconstruction de plusieurs radicaux étymologiques -230

Table 4.6 Analyse constructionnelle des 42 dérivés déverbaux protoromans en

*/-'ur-a/ 232

Table 4.7 Analyse constructionnelle des dix dérivés en */-'ur-a/ de verbes appartenant à la flexion en */-'a-/ 233

Table 4.8 Analyse constructionnelle des trois dérivés en */-'ur-a/ de verbes appartenant à la flexion en */-'i-/ 233

Table 4.9 Analyse constructionnelle des quatre dérivés en */-'ur-a/ de verbes appartenant à la flexion en */-'e-/ 234

Table 4.10 Analyse constructionnelle des 25 dérivés en */-'ur-a/ de verbes appartenant à la flexion en */'-e-/ 234

Table 4.11 Dérivés monosémiques en */-'ur-a/ 242

Table 4.12 Dérivés polysémiques en */-'ur-a/ $\mathbf{2} \mathbf{2 4 2}$

Table 4.13 Tableau comparatif des types de sens présentés par les lexèmes portant le suffixe */-'ur-a/ 246

Table 4.14 Répartition des types de sens pour les monosèmes - 246

Table 4.15 Répartition des polysèmes à deux sens - 247

Table 4.16 Répartition des polysèmes à trois sens $-\mathbf{2 4 7}$

Table 4.17 Analyse stratigraphique des dérivés en */-'ur-a/ -257

Table 4.18 Analyse de la variation stratigraphique à l'intérieur des différents types phonologiques et sémantiques des dérivés en */-'ur-a/ selon la reconstruction comparée (I) 259

Table 4.19 Analyse de la variation stratigraphique à l'intérieur des différents types phonologiques et sémantiques des dérivés en */-'ur-a/ selon la reconstruction comparée (II) $-\mathbf{2 6 0}$

Table 4.20 Dérivés en */-'ur-a/ attribuables à des variétés régionales du protoroman -261

Table 4.21 Unités lexico-phonologiques et lexico-sémantiques reconstructibles seulement pour des aires géographiques restreintes -261

Table 4.22 Dérivés protoromans en */-'ur-a/ attribuables à des variétés d'immédiat communicatif en raison de la date tardive de l'apparition de leur corrélat en latin écrit -264 
